\title{
Synapsin I Senses Membrane Curvature by an Amphipathic Lipid Packing Sensor Motif
}

\author{
Ludwig Krabben, ${ }^{1}$ Anna Fassio, ${ }^{2 \star}$ Vikram Kjøller Bhatia, ${ }^{4 \star}$ Arndt Pechstein, ${ }^{1,5 *}$ Franco Onofri, ${ }^{2 \star}$ Manuela Fadda, ${ }^{2,3}$ \\ Mirko Messa, ${ }^{2}$ Yijian Rao, ${ }^{1}$ Oleg Shupliakov, ${ }^{5}$ Dimitrios Stamou, ${ }_{4}^{4}$ Fabio Benfenati, ${ }^{2,3}$ and Volker Haucke ${ }^{1,6}$ \\ ${ }^{1}$ Institute of Chemistry and Biochemistry and Neurocure Cluster of Excellence, Freie Universität, 14195 Berlin, Germany, ${ }^{2}$ Department of Experimental \\ Medicine, University of Genova and Instituto Nazionale di Neuroscienze, 16132 Genova, Italy, ${ }^{3}$ Department of Neuroscience and Brain Technologies, Italian \\ Institute of Technology, 16163 Genova, Italy, ${ }^{4}$ University of Copenhagen, Faculty of Health Sciences, Department of Neuroscience and Pharmacology and \\ Nano-Science Center, 2100 Copenhagen, Denmark, ${ }^{5}$ Department of Neuroscience, Developmental Biology for Regenerative Medicine, Karolinska Institutet, \\ 17177 Stockholm, Sweden, and ${ }^{\circ}$ Leibniz-Institut für Molekulare Pharmakologie, 13125 Berlin, Germany
}

Sustained neurotransmitter release at synapses during high-frequency synaptic activity involves the mobilization of synaptic vesicles (SVs) from the tightly clustered reserve pool (RP). Synapsin I (Syn I), a brain-specific peripheral membrane protein that undergoes activity-dependent cycles of SV association and dissociation, is implicated in RP organization via its ability to cluster SVs. Although Syn I has affinity for phospholipids, the mechanism for the reversible association of synapsin with SV membranes remains enigmatic. Here, we show that rat Syn I is able to sense membrane curvature via an evolutionary conserved amphipathic lipid packing sensor motif(ALPS). Deletion or mutational inactivation of the ALPS impairs the ability of Syn I to associate with highly curved membranes and with SVs. Furthermore, a Syn I mutant lacking ALPS displays defects in its ability to undergo activity-induced cycles of dispersion and reclustering in neurons and fails to induce vesicle clustering in vitro. Our data suggest a crucial role for ALPS-mediated sensing of membrane curvature in regulating synapsin function.

\section{Introduction}

Neurotransmission requires the exo-endocytic cycling of small (40 nm) synaptic vesicles (SVs) (Murthy and De Camilli, 2003). While the readily releasable pool of SVs resides at or near active zones, a distantly located, tightly clustered reserve pool (RP) of SVs is required for sustained neurotransmitter release during high-level activity (Rizzoli and Betz, 2005). The organization of the RP of SVs is linked to the peripheral SV-associated phosphoprotein synapsin I (Syn I) (Cesca et al., 2010; Shupliakov et al., 2011).

Synapsins (I-III) are evolutionary conserved neuron-specific proteins (Cesca et al., 2010). Syn I associates with SVs, partly via binding to acidic phospholipids (Benfenati et al., 1993), and represents a major component of purified SVs (Takamori et al., 2006). Syn I is surface-active, covers a large proportion of the SV surface, and promotes clustering of SVs within the RP (Benfenati

Received Aug. 24, 2011; revised 0ct. 10, 2011; accepted 0ct. 25, 2011.

Author contributions: L.K., A.P., O.S., D.S., F.B., and V.H. designed research; L.K., A.F., V.K.B., A.P., F.O., M.F., M.M., and Y.R. performed research; L.K., A.F., V.K.B., A.P., M.M., D.S., and F.B. analyzed data; F.B. and V.H. wrote the paper.

This work was supported by grants from the Italian Ministry of University and Research, Compagnia di San Paolo (Torino) and Telethon-Italy (to F.B.), EU-FP7 [HEALTH-F2-2009-242167 (SynSys) to 0.S.], the Swedish Research Council (0.S., A.P.), and the DFG (Exc-257; SFB958/A01 to V.H.).We thank Drs. Enrico Defranchi and Silvia Casagrande for experimental help.

*A.F., V.K.B., A.P., and F.O. contributed equally to this work.

Correspondence should be addressed to Volker Haucke, Institute of Chemistry and Biochemistry and Neurocure Cluster of Excellence, Freie Universität, Takustrasse 6, 14195 Berlin, Germany. E-mail: volker.haucke@fu-berlin.de. DOI:10.1523/JNEUROSCI.4345-11.2011

Copyright $\odot 2011$ the authors $\quad 0270-6474 / 11 / 3118149-06 \$ 15.00 / 0$ et al., 1993; Pieribone et al., 1995; Rosahl et al., 1995; Ryan et al., 1996).

Syn I dissociates from SVs upon stimulation, allowing mobilization of SVs from the RP during activity (Hosaka et al., 1999; Chi et al., 2001; Menegon et al., 2006; Cesca et al., 2010). After cessation of the stimulus, Syn I reassociates with SVs, promoting their reclustering within the RP (Chi et al., 2001; Menegon et al., 2006). The mechanisms that attach Syn I to SVs during this functional cycle remain enigmatic. Syn I-binding proteins such as CaMKII or Src are not exclusively associated with SVs and are present in insufficient copy numbers (Cesca et al., 2010). Syn I also directly and saturably associates with (Benfenati et al., 1989) and clusters (Benfenati et al., 1993) acidic liposomes. Because of the broad lipid specificity, this interaction likewise appears insufficient to explain the selective association of synapsins with SVs at synapses.

Amphipathic lipid packing sensor (ALPS) motifs able to insert into curved membranes via an amphipathic helix rich in hydrophilic polar amino acids (Bigay et al., 2005; Antonny, 2011) have been identified in several proteins (Drin et al., 2007), including putative motifs in Syn II and Syn III. Here, we show that the major synapsin isoform, Syn I, contains an ALPS motif that, by sensing membrane curvature (MC), facilitates Syn I association with SVs and regulates its vesicle clustering activity. A Syn I mutant lacking the ALPS displays defects in its ability to associate with highly curved membranes, including SVs, and to undergo activity-induced cycles of dispersion and reclustering in primary neurons. Moreover, mutant Syn I is partially defective in facilitating vesicle clustering in vitro. These data argue for a crucial role 


\section{A}

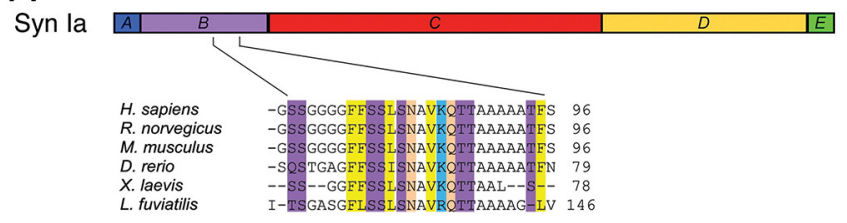

Syn Ila

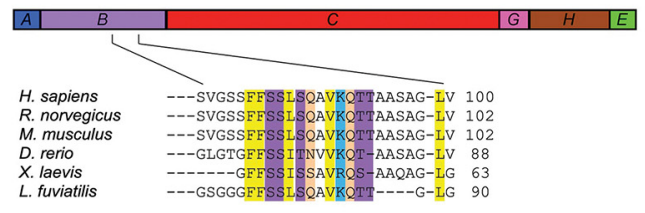

Syn IIla

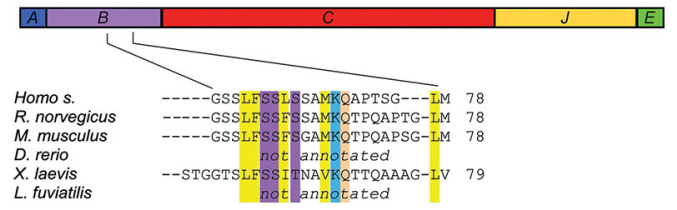

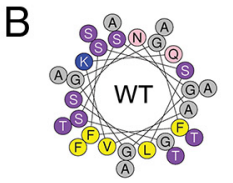
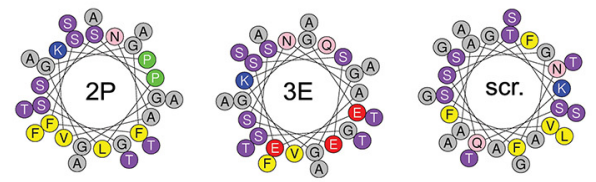

C

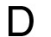

E
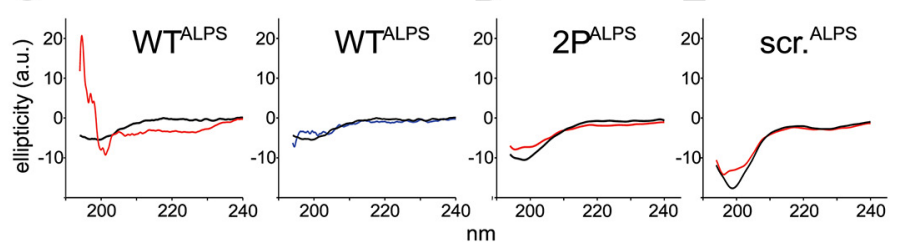

Figure 1. An ALPS motif within the B domain of Syn I. A, Schematics of rat Syn la, Syn Ila, and Syn Illa indicating the putative ALPS. Multiple sequence alignment of putative ALPS within Syn orthologues from different species. $\boldsymbol{B}$, Helical wheel projection of Syn la-derived ALPS peptide. Purple, polar hydrophilic residues; yellow, hydrophobic. C-E, CD spectra of Syn I-derived ALPS peptides in the absence (black) or presence of $30-100 \mathrm{~nm}$ (red) or $400 \mathrm{~nm}$ (blue) liposomes. C, WT ALPS peptide. D, 2P-ALPS inactive mutant. E, Scrambled peptide.

for ALPS-mediated sensing of MC in regulating synapsin function.

\section{Materials and Methods}

Peptides and antibodies. Peptide sequences were as follows: rat Syn Ia WT $^{\text {ALPS }}$ (amino acids 69-96): GSSGGGGFFSSLSNAVKQTTAAAAATF S); $2 P^{\text {ALPS }}$ : GSSGGGGFFSSLSNAVKPTTAAAAATFS; $3 E^{\text {ALPS }}$ : GSSGGG GEESSESNAVKQTTAAAAATFS; scrambled ${ }^{\text {ALPS }}$ : VAANFSTSQSGAFG KASFLAATGGSSTG; Kes1p (7-29, yeast): SSSWTSFLKSIASFNGDLSS LSA; AP180 (753-70, human): LGSDLSSLASLVGNLGI.

For single liposome assays, peptides were $N$-terminally tagged with CAAP. Antibodies used were Syn I, bassoon (Synaptic Systems), synaptophysin (Sigma-Aldrich), and GFP (Stressgen).

Circular dichroism spectroscopy. Peptides (3 mM) were dialyzed into circular dichroism (CD) buffer (10 mM Tris, pH 7.5, $150 \mathrm{~mm} \mathrm{NaF}$ ) and measured on a Jasco J-810 spectrometer at $20^{\circ} \mathrm{C}$. Spectra represent averages of 10 scans recorded from 194 to $240 \mathrm{~nm}$. Control spectra were subtracted.

Plasmids, generation of stable cell lines, protein purification. eGFP-Syn Ia was purified from stably transfected doxycycline-inducible HEK293 cells. Pellets were resuspended in $20 \mathrm{~mm}$ HEPES, pH 7.4, $100 \mathrm{~mm} \mathrm{NaCl}$, $50 \mathrm{~mm} \mathrm{KCl}, 5 \mathrm{~mm} \mathrm{MgCl} 2,5 \mathrm{~mm}$ DTT, 0.3\% CHAPS plus protease inhibitors, cracked and diluted with one volume of $10 \mathrm{~mm}$ HEPES, pH 7.4, 150 $\mathrm{mm} \mathrm{NaCl}, 2$ mm DTT. Cleared supernatant was applied to GFP-TrapA columns, washed, eluted ( $0.2 \mathrm{M}$ glycine, $\mathrm{pH} 2.5)$, and neutralized. Purity was assessed by measuring (A260/A280) and silver-stained SDS-PAGE.

Single liposome curvature assays. Single liposome binding assays were done as in Bhatia et al. (2009) with liposomes of a SV-like composition [69\% DOPC (1,2-dioleoyl-sn-Glycero-3-Phosphocholine), 30\% DOPS (1,2-dioleoyl-sn-glycero-3-phospho-L-serine), 0.5\% DOPE-Biotin (1,2Dioleoyl-sn-Glycero-3-Phosphoethanolamine- $N$-(Cap Biotinyl)), 0.5\% DID (1,10-dioctadecyl-3,3,30,30-tetramethyl-indodicarbocyanine perchlorate); Invitrogen]. For control experiments using eGFP-PH, liposomes contained $5 \% \mathrm{PI}(4,5) \mathrm{P}_{2}$ at the expense of DOPS. Samples were analyzed as described previously (Bhatia et al., 2009).

Analysis of Syn I-induced liposome clustering. SV-like small liposomes $(\varnothing 60 \mathrm{~nm}$ ) [phosphatidylcholine (PC):phosphatidylethanolamine (PE): phosphatidylserine (PS):phosphatidylinositol (PI):cholesterol $=27: 30$ : $10: 3: 30 ; 1 \%$ rhodamine-PE] were prepared by dehydration/rehydration in HEPES-buffered saline (HBS) (20 mm HEPES, pH 7.4, $200 \mathrm{~mm} \mathrm{NaCl}$ ) followed by sonication in HBS for 10 min under argon flow while cooling. Precleared liposomes were incubated in HBS with or without $100 \mu \mathrm{M}$ peptide $\left(3 \mathrm{~min}, 37^{\circ} \mathrm{C}\right.$ ) before addition of $75 \mathrm{~nm}$ eGFP-Syn I (WT or $\Delta$ ). Samples incubated for $10 \mathrm{~min}$ at $37^{\circ} \mathrm{C}$ were imaged by fluorescence microscopy and quantitatively analyzed using Volocity (Improvision). Mean particle sizes were determined from $>10$ fields of view $(n=2$ independent experiments). Data were analyzed statistically using a oneway ANOVA with Bonferoni's multiple comparison as a post-test.

Liposome and SV binding. Synapsin-depleted SVs (10 $\mu \mathrm{g})$ (Schiebler et al., 1986) or sucrose-loaded liposomes (PC:PE:PS:PI:cholesterol $=27$ : 30:10:3:30) (Benfenati et al., 1989) were incubated for $1 \mathrm{~h}$ at $4^{\circ} \mathrm{C}$ with wild-type (WT) or mutant eGFP-Syn I (40-200 nм) in $220 \mathrm{~mm}$ glycine, $30 \mathrm{~mm} \mathrm{NaCl}, 5 \mathrm{~mm}$ Tris-HCl, $4 \mathrm{~mm}$ HEPES, $\mathrm{pH}$ 7.4, $0.22 \mathrm{~mm} \mathrm{NaN}_{3}$, and $100 \mu \mathrm{g} / \mathrm{ml} \mathrm{BSA}$. SVs or liposomes were reisolated by ultracentrifugation $(400,000 \times \mathrm{g}, 30 \mathrm{~min})$ through a $10 \%(\mathrm{w} / \mathrm{w})$ sucrose cushion. Samples were immunoblotted and recovery of ${ }^{14} \mathrm{C}$-PC was measured.

Optical recordings in primary neurons. Hippocampal neurons from E17-E18 mouse embryos of either sex were transfected (11-14 DIV) with lipofectamine 2000, fixed (4\% PFA 4 d posttransfection), and immunostained. Optical recordings ( $1 \mathrm{~Hz}$ for $180 \mathrm{~s}$ ) were performed at 15-18 DIV (3-5 d posttransfection) as described previously (Menegon et al., 2006). Fluorescence changes of eGFP-Syn Ia at synaptic boutons were normalized to the starting fluorescence $\left(\mathrm{F}_{0}\right)$. Time constants and plateaus for dispersion and reassociation were determined by fitting the respective curves with a single exponential function.

\section{Results}

\section{An ALPS within the B domain of Syn I}

BLAST searches for presynaptic proteins that contain potential ALPSs (Drin et al., 2007) identified Syn Ia/b, a protein known to reversibly associate with small SVs (Hosaka et al., 1999; Chi et al., 2001), i.e., high curvature membranes (Fig. 1A). The putative ALPS within mammalian Syn Ia corresponds to amino acids 69-96 belonging to its domain B and is rich in polar hydrophilic and hydrophobic residues (Fig. $1 A, B$ ). Sequences with similar features are found within rat or human Syn I, Syn II, and Syn III and within Syn I from lamprey and Xenopus, suggesting that the putative ALPS is evolutionary conserved (Fig. $1 A$ ). When plotted in a helical wheel, this motif can fold into an amphipathic $\alpha$-helix (Fig. $1 B$ ).

ALPSs adopt a random conformation in solution, but fold into $\alpha$-helical structure when contacting curved lipid membranes 
A
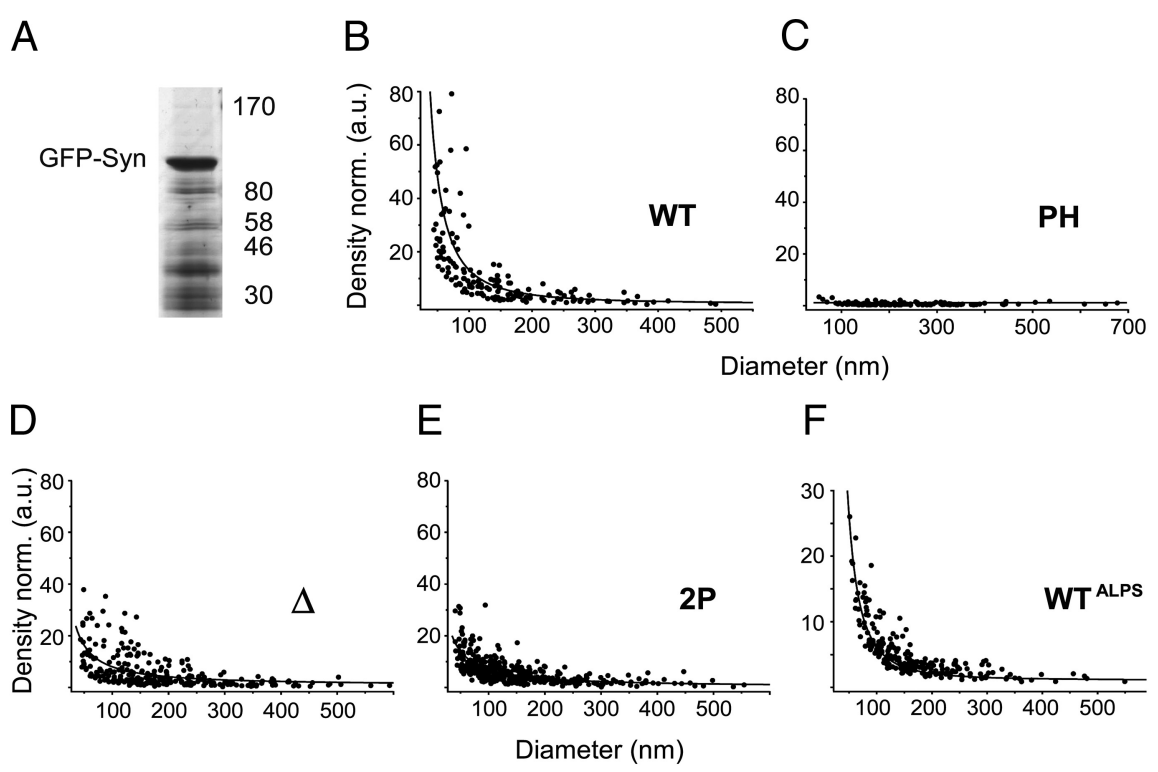

$\mathrm{F}$

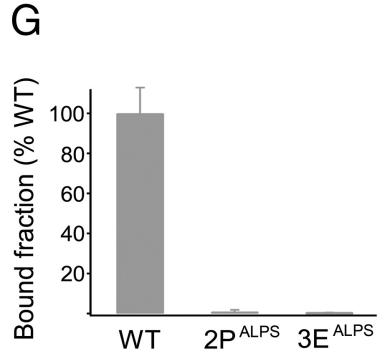

$\mathrm{H}$

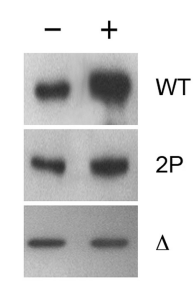

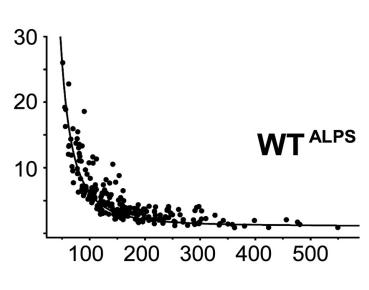

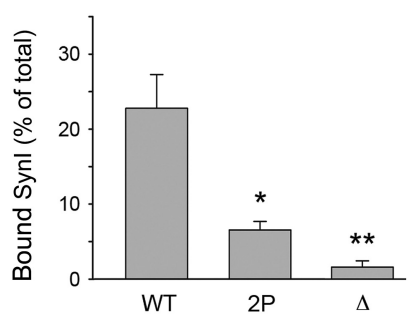

Figure 2. Membrane curvature sensing and liposome binding by Syn I involves an ALPS. A, Silver-stained gel illustrating purified eGFP-Syn la. Right, Molecular weight markers in $\mathrm{KDa}$. $\boldsymbol{B}-\boldsymbol{F}$, Single liposome curvature assays. A mixture of DID-labeled fluorescent liposomes of various sizes $(\emptyset 50-700 \mathrm{~nm})$ immobilized on a glass support were incubated with proteins or peptides: WT-eGFP-Syn I (B); the pleckstrin homology domain of phospholipase ( $\delta$ (curvature-insensitive) (eGFP-PH $\left.{ }^{\text {PLC } \delta} ; \boldsymbol{C}\right) ; \triangle$ ALPS-eGFPSyn I (D); 2P-ALPS-eGFP-Syn I (E); Atto-labeled Syn I-derived ALPS peptide (WT $\left.{ }^{\text {ALPS }} ; \boldsymbol{F}\right)$. Plots display protein/peptide density (arbitrary units, a.u.) versus liposome diameter ( $\emptyset$ range: $50-700 \mathrm{~nm}$ ). G, Liposome association of WT ${ }^{\text {ALPS }}$ and of $2 P^{\text {ALPS }}$ or $3 E^{\text {ALPS }}$ mutant peptides expressed as a percentage of the input peptide. $\boldsymbol{H}$, Binding of WT or ALPS mutants of Syn I (50 nM) to SV-like liposomes ( $\emptyset 50-70 \mathrm{~nm} ; 20 \mu \mathrm{g}$ of phospholipid/sample). Bound Syn I (left) is expressed in percentage of total Syn I added (mean $\pm \mathrm{SEM}) .{ }^{*} p<0.05 ;{ }^{* *} p<0.01$ versus WT, Dunnett's multiple-comparison test (right).

(Drin et al., 2007). To test whether the putative ALPS within Syn I is able to sense MC, we incubated a peptide corresponding to amino acids 69-96 of rat Syn I (WT ${ }^{\text {ALPS }}$ ) with liposomes of different diameters and analyzed its secondary structure by circular dichroism (CD) spectroscopy. In solution, the Syn I-derived peptide displayed a random conformation (Fig. 1C, black line). The addition of small, high-curvature liposomes, similar in size to SVs (i.e., $30-50 \mathrm{~nm}$ ), produced a CD spectrum containing features of $\alpha$-helical (negative ellipticity $\sim 202 \mathrm{~nm}$ and a strong increase in positive ellipticity at $195 \mathrm{~nm}$ ) and random coil conformation (Fig. 1C, red line). Simulation of the CD spectrum predicted $\sim 30 \% \alpha$-helical content, consistent with a weak association of ALPS with membranes (Antonny, 2011). The CD spectrum therefore likely represents a weighted average of vesicle-bound and free soluble Syn I-derived peptide. No induction of helix formation was observed with $400 \mathrm{~nm}$ liposomes (Fig. 1C, blue line), indicating that the Syn I-derived peptide indeed is able to sense MC.

To corroborate this, we synthesized a peptide in which S78 and Q86 were exchanged for helix-breaking proline residues $\left(2 \mathrm{P}^{\mathrm{ALPS}}\right)$ and a scrambled version of the Syn I-derived ALPS peptide (scr). $2 \mathrm{P}^{\mathrm{ALPS}}$ and scr appeared unstructured in CD with or without high-curvature liposomes (Fig. 1D,E). Both peptides thus lack the ability to undergo highcurvature membrane-induced $\alpha$-helix formation.

\section{Membrane curvature sensing by Syn I involves an ALPS motif}

The above data indicate that the Syn I-derived ALPS peptide is able to sense MC. To test whether full-length Syn Ia can sense MC, we purified recombinant eGFP-Syn Ia from stably transfected HEK293 cells (Fig. 2A). eGFP-Syn Ia was soluble and able to bind and cluster liposomes similar to native Syn I (Benfenati et al., 1993) (see below). The ability of eGFPSyn I or of Syn I-derived ALPS peptides to sense MC was then analyzed using quantitative fluorescence imaging of single liposomes with various diameters and MC (Bhatia et al., 2009). The pleckstrin homology domain $(\mathrm{PH})$ of phospholipase $\mathrm{C} \delta$, a phosphatidylinositol 4,5-bisphosphate binding domain unable to sense curvature (Bhatia et al., 2009), served as the negative control. Quantitative confocal imaging was used to record the fluorescence intensity of eGFP-tagged proteins colocalized with DID-labeled liposomes of various sizes. Plots of the relative density of bound eGFP-Syn Ia versus liposome size revealed a remarkable preference of WT eGFP-Syn I for liposomes with a diameter $<100 \mathrm{~nm}$, indicative of its ability to sense MC (Fig. 2B), whereas eGFP-PH bound equally well to liposomes of all sizes (Fig. 2C). Two mutant variants of Syn I in which the putative curvature-sensing ALPS was either deleted ( $\triangle$ ALPS) or mutationally inactivated (2PALPS) showed a greatly reduced ability to associate with high-curvature liposomal membranes (Fig. $2 D, E)$ or with small SV-like membrane vesicles mimicking size and phospholipid composition of native SVs (Fig. $2 H$ ).

The fluorescently labeled Syn I-derived ALPS peptide fully retained the ability to sense MC, similar to holo-Syn I (Fig. 2 F). The $2 \mathrm{P}^{\text {ALPS }}$ peptide or a peptide carrying substitutions in the hydrophobic face of the $\alpha$-helix (F76/F77/L80 to E) failed to sense $\mathrm{MC}$, resulting in their inability to stably associate with liposomal membranes (Fig. 2G).

The limited residual curvature-sensing ability of $\triangle$ ALPS- and 2P-ALPS mutant Syn I (Fig. 2D,E) may be explained by other potential curvature-sensitive elements not encoded within the identified ALPS. In line with this, multiple determinants within domains B, C, and E cooperate in targeting Syn I to presynaptic membranes (Gitler et al., 2004). Our data indicate that curvature sensing by Syn I largely depends on an ALPS within its B domain.

\section{Syn I-induced vesicle clustering modulated by} ALPS-mediated membrane curvature sensing

A central function of Syn I is to organize the RP of SVs via its ability to cluster small vesicles (Cesca et al., 2010). Syn I-mediated liposome binding and clustering is saturable (Benfenati et al., 1993) and depends on Syn I oligomerization via C- and 
A

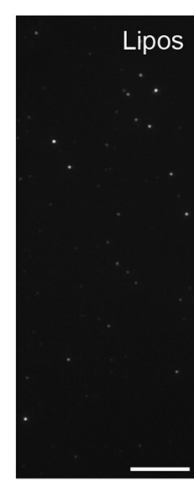

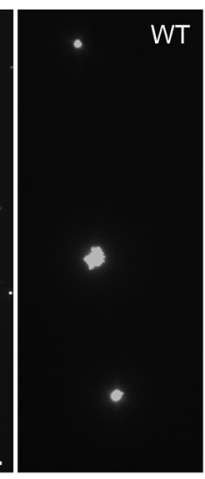

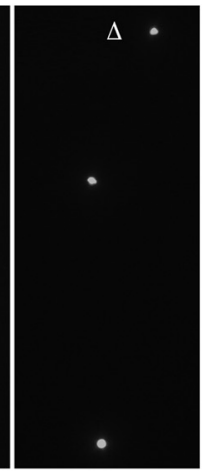

C

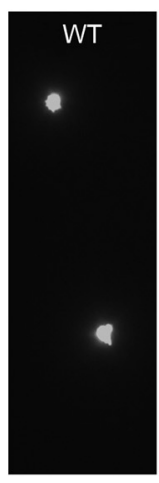

peptide competition

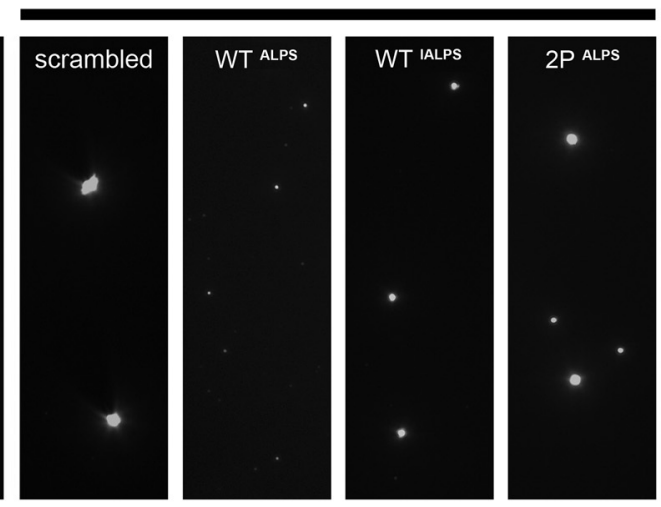

B

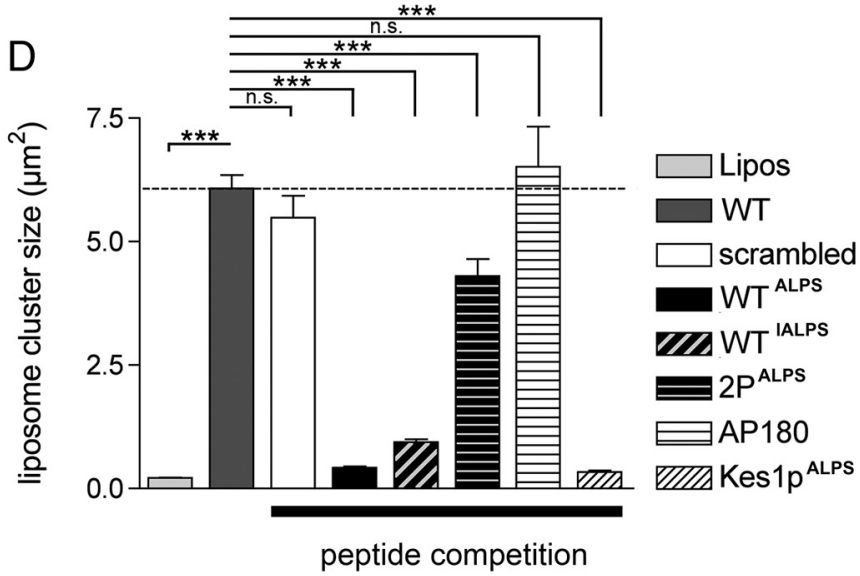

Figure 3. Syn I-mediated vesicle clustering is modulated by ALPS-mediated membrane curvature sensing. $\boldsymbol{A}$, Representative images of liposome (Lipos) clusters induced by WT or mutant eGFP-Syn la $(\Delta)$. B, Quantitative analysis of data in $\boldsymbol{A}$. C, Same as $\boldsymbol{A}$, but liposomes were preincubated with peptides (100 $\mu$ M). WT ${ }^{\text {ALPS }}$, ALPS peptide from human or rat Syn la; WT ${ }^{\text {IALPS }}$, ALPS peptide from lamprey Syn I; $2 P^{\text {ALPS }}$, proline-containing mutant version of WT ${ }^{\text {ALPS }}$; AP180, control peptide from AP180; Kes1p ${ }^{\text {ALPS }}$, ALPS peptide from yeast Kes1p. D, Quantifications of data in C. Scale bar, $10 \mu \mathrm{m} .{ }^{* * *} p<0.001$ (one-way ANOVA); n.s., non-significant.

E-domain-mediated interactions (Hosaka and Südhof, 1999; Monaldi et al., 2010). To address the role of ALPS-mediated curvature sensing in synapsin function, we monitored the ability of WT and ALPS mutant Syn I to cluster liposomes mimicking the size and lipid composition of native SVs in vitro. Purified WT or mutant eGFP-Syn Ia ( $\Delta$ ALPS) was incubated with fluorescently labeled SV-like liposomes and analyzed by fluorescence microscopy. Addition of WT eGFP-Syn I clustered small liposomes into larger assemblies. Mutant eGFP-Syn I ( $\Delta$ ALPS) lacking its ALPS showed a significantly reduced clustering ability (Fig. $3 A, B$ ), suggesting that ALPS-mediated curvature sensing modulates the ability of Syn I to cluster high curvature phospholipid vesicles. In further support of this, addition of ALPS peptides derived from mammalian (WT ${ }^{\text {ALPS }}$ ) or lamprey (WT ${ }^{\text {IALPS }}$ ) Syn I potently inhibited Syn Ia-induced liposome clustering, whereas a mutant version of the ALPS peptide unable to sense curvature $\left(2 \mathrm{P}^{\mathrm{ALPS}}\right.$; compare Fig. 2) showed much-reduced activity (Fig. 3C,D). Vesicle clustering was also inhibited by a known ALPS peptide derived from Kes1p (Drin et al., 2007), but not by a control peptide taken from AP180. Thus, the ability to sense MC is critical for peptide competition of Syn I-induced liposome clustering.

\section{Membrane curvature sensing by its ALPS modulates Syn I} dispersion and reassociation with SV membranes

Syn I reversibly binds to small SVs and undergoes activitydependent association/dissociation cycles (Cesca et al., 2010). It is thus conceivable that MC sensing participates in the ability of synapsin to reassociate with SVs following its axonal dispersion. To study the role of ALPS-mediated curvature sensing in regulating SV binding, highly purified rat SVs depleted of endogenous synapsin (Schiebler et al., 1986) were incubated with WT eGFPSyn I or mutants, in which the ALPS was either deleted ( $\triangle$ ALPS) or inactivated (2P-ALPS). While WT-Syn I avidly bound to SVs in a saturable and concentration-dependent manner, 2P-ALPSSyn I displayed a strongly reduced SV binding ability. The $\triangle$ ALPS-mutant virtually failed to associate with SV membranes (Fig. $4 A, B$ ). Hence, the ability of Syn I to associate with native SVs strongly depends on the presence of an intact ALPS.

Syn I association with SVs is critically involved in its activityinduced axonal dispersion and presynaptic reclustering, a function linked to the organization of the SV RP (Chi et al., 2001; Menegon et al., 2006). To assess the role of Syn I-mediated curvature sensing via its ALPS during synaptic activity, hippocampal neurons expressing WT- or $\Delta$ ALPS-eGFP-Syn I were analyzed by live imaging. No gross differences in the overall expression of WT and mutant Syn I were observed (Fig. 4D, left). eGFP-Syn Ia (WT) exhibited a punctate localization corresponding to presynaptic boutons positive for the active zone marker bassoon (Fig. $4 C)$. A similar, although significantly less intense, presynaptic staining was seen for $\triangle$ ALPS-eGFP-Syn I (Fig. $4 D$, right). Thus, ALPS-mediated curvature sensing is not essential for presynaptic targeting of Syn I, although it improves targeting efficacy.

Synaptic activity induces the axonal dispersion of Syn I, reflecting its phosphorylation-induced dissociation from SV mem- 

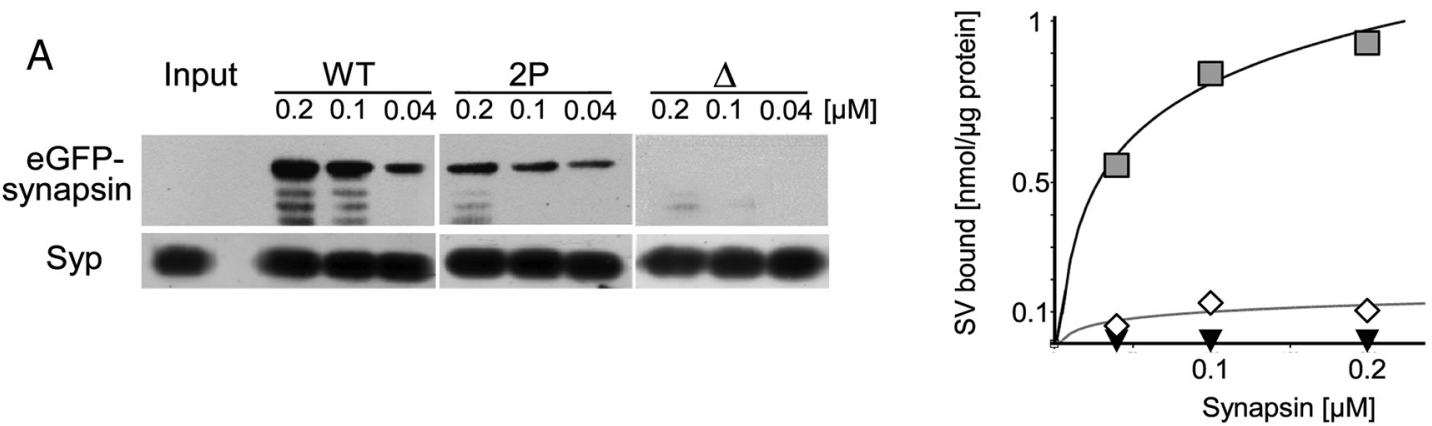

B

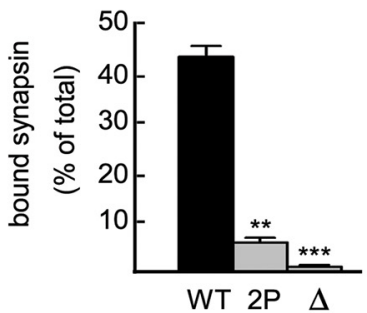

D

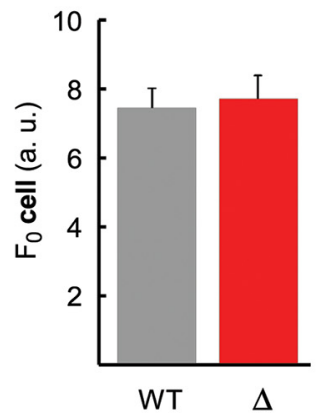

C

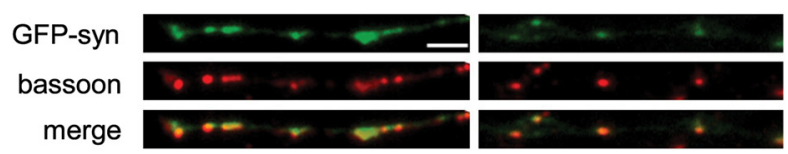

F

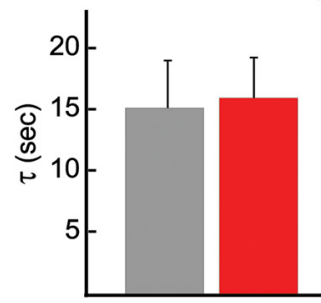

WT

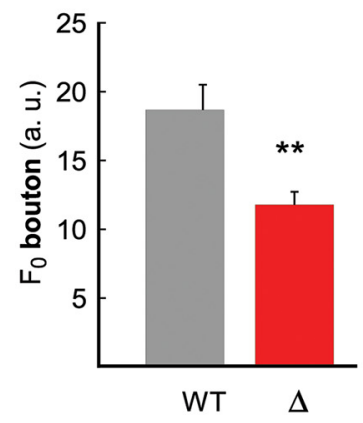

Dispersion

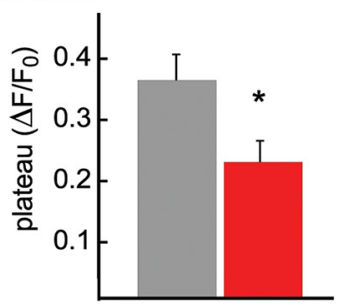

WT

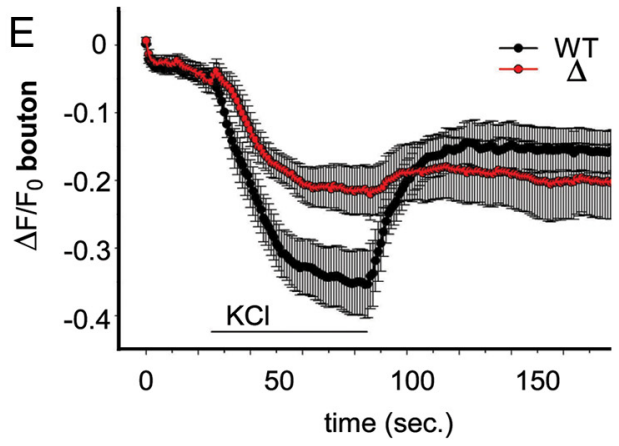

G

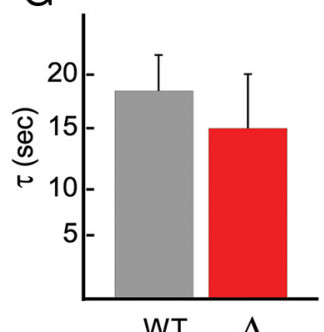

Recovery

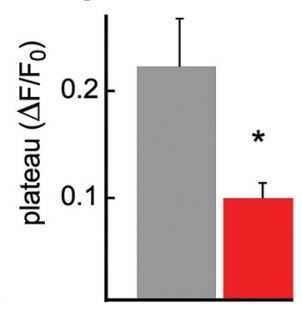

WT $\Delta$

$\mathrm{H}$

\begin{tabular}{|c|c|c|c|c|c|}
\hline SYNI/II/III & \multirow{2}{*}{$\begin{array}{c}\mathrm{F}_{0} / \text { bouton } \\
\text { background }\end{array}$} & (a.u.) & \multicolumn{2}{|c|}{ DISPERSION } & \multicolumn{2}{|c|}{ RECOVERY } \\
\cline { 3 - 6 } & & $\tau(\mathrm{sec})$ & Plateau $\left(\Delta \mathrm{F} / \mathrm{F}_{0}\right)$ & $\tau(\mathrm{sec})$ & Plateau $\left(\Delta \mathrm{F} / \mathrm{F}_{0}\right)$ \\
\hline WT-Syn I & $18.01 \pm 1.61$ & $14.9 \pm 3.3$ & $0.45 \pm 0.11$ & $17.4 \pm 6.0$ & $0.30 \pm 0.09$ \\
\hline$\Delta$ ALPS-Syn I & $10.70 \pm 1.80^{* *}$ & $20.6 \pm 5.3^{\mathrm{NS}}$ & $0.30 \pm 0.02^{*}$ & $16.6 \pm 5.3^{\mathrm{NS}}$ & $0.20 \pm 0.04^{\mathrm{NS}}$ \\
\hline
\end{tabular}

Figure 4. Membrane curvature sensing by ALPS modulates Syn I dispersion and SV reassociation. $A, B$, Increasing concentrations (40-200 nm) of WT-eGFP-Syn I (squares), 2P-ALPS-eGFP-Syn I (diamonds), or $\triangle$ ALPS-eGFP-Syn I (triangles) incubated with synapsin-depleted SVs. Reisolated SVs were immunblotted for eGFP-Syn la or synaptophysin (Syp). $A$, Representative immunoblot and quantitation of densitometric scans fitted with a one-site binding function. $B$, Percentage of bound eGFP-Syn I (mean \pm SEM; $n=4$ independent experiments; ${ }^{* *} p<0.01 ;{ }^{* * *} p<0.001$ vs WT, Dunnett's multiple-comparison test). C, Representative images illustrating presynaptic localization of eGFP-Syn I (WT or $\triangle$ ALPS, green). Boutons were labeled with bassoon (red). Scale bar, $2 \mu \mathrm{m}$. $D$, Reduced presynaptic expression of eGFP-Syn la ( $\Delta$ ALPS). Left, Expression level of WT and $\Delta$ ALPS eGFP-Syn la quantified by total GFP fluorescence (mean \pm SEM; $n=2$ independent experiments). Right, Presynaptic expression measured by averaging basal fluorescence $\left(\mathrm{F}_{0}\right)$ in 10 frames preceding stimulation (mean $\pm S E M ; 9$ coverslips/construct; 20 boutons/coverslip; $n=2$ independent experiments; ${ }^{* *} p<0.01$, Student's $t$ test). $\boldsymbol{E}$, Time courses of changes in eGFP fluorescence before, during, and after stimulation (black horizontal bar) in neurons expressing eGFP-Syn la (black dots) or eGFP-Syn la ( $\triangle$ ALPS; red dots) (mean \pm SEM) $(9$ coverslips/construct; 20 boutons/coverslip; $n=2$ independent experiments). $\boldsymbol{F}$, Rate of dispersion ( $\tau$; left) during stimulation fitted to single exponential. The dispersion plateau $\left(\Delta \mathrm{F} / \mathrm{F}_{0}\right.$; right) was significantly lower for $\Delta \mathrm{ALPS}$ compared with WT $\left({ }^{*} p<0.05\right.$, Student's $t$ test). $G$, Rate of reassociation $(\tau ;$ left) from the end of the stimulation to the end of the recording fitted to single exponential. The amplitude of recovery (i.e., the $\Delta F / F_{0}$ plateau; right) was significantly reduced for $\Delta A L P S\left({ }^{*} p<0.05\right.$, Student's $t$ test). Reassociation kinetics (left) were unaffected. $\boldsymbol{H}$, Depolarization-induced dispersion and recovery of WT- and $\Delta$ ALPS-Syn I in nerve terminals of Synl/II/III ${ }^{-I-}$ neurons (mean \pm SEM; $n=5-6$ experiments). ${ }^{*} p<0.05 ;{ }^{* *} p<0.01$; Student's $t$ test; NS, non-significant. 
branes. As the stimulus ceases, Syn I reclusters within presynaptic boutons due to its rebinding to small SVs (Chi et al., 2001; Cesca et al., 2010). We monitored the distribution of eGFP-Syn I by optically recording fluorescence changes elicited by neuronal activity within presynaptic boutons. Chemical stimulation with $\mathrm{KCl}$ caused a loss of fluorescence intensity of presynaptic eGFPSyn I with a half-time $(\tau)$ of $\sim 15 \mathrm{~s}$, similar to Chi et al. (2001) (Fig. $4 E, F$ ), reflecting its axonal dispersion. Following cessation of the stimulus, eGFP-Syn I fluorescence recovered with a $\tau$ of $18-20$ s to reach $\sim 80 \%$ of its initial value (Fig. $4 E, G$ ). A much less pronounced activity-induced dispersion was observed for $\Delta$ ALPS-eGFP-Syn I $\left(\Delta \mathrm{F} / \mathrm{F}_{0}\right.$; Fig. $\left.4 E, F\right)$. Dispersion kinetics were, however, indistinguishable between WT and mutant synapsin $(\tau$ dispersion; Fig. $4 F$ ). Importantly, $\Delta$ ALPS-eGFP-Syn I displayed a severely reduced ability to recluster within presynaptic boutons $\left(\mathrm{DF} / \mathrm{F}_{0}\right.$; Fig. $\left.4 G\right)$, although the rate constant of recovery remained nearly unchanged (Fig. $4 G$; $t$ recovery). Reduced presynaptic targeting and impaired activity-induced dispersion of $\Delta$ ALPS-Syn I were also seen in neurons from Syn I/II/III ${ }^{-/-/-}$mice (Fig. $4 H$ ), indicating that these phenotypes do not reflect differential association of expressed WT versus mutant Syn I with endogenous synapsins. However, recovery of $\Delta$ ALPS-Syn I was only slightly and nonsignificantly impaired compared to WT-Syn I, suggesting that the absence of competition by endogenous ALPScontaining synapsins might facilitate reassociation with SVs. Thus, the ability of Syn I to sense MC via its ALPS critically modulates the amplitude of responses, but not the kinetics of the activity-induced dispersion and reclustering. These observations are consistent with the strongly reduced ability of mutant Syn I to bind to SVs (Fig. $4 A, B$ ).

\section{Discussion}

We have identified and functionally characterized an evolutionary conserved ALPS within the SV-associated protein Syn I. Our data indicate that ALPS-mediated curvature sensing is required for the ability of Syn I to selectively associate with small phospholipid vesicles and native SVs. Furthermore, we demonstrate a crucial functional role for ALPS-mediated recognition of $\mathrm{MC}$ in regulating the ability of Syn I to induce vesicle clusters in vitro and to reversibly associate with SVs in live neurons. Our data provide a mechanistic explanation for the selective association of Syn I with small SVs (Takamori et al., 2006; Cesca et al., 2010) and suggest a potential role for MC sensing by Syn I in presynaptic membrane organization. Induction and sensing of MC by exo-endocytic proteins is a key mechanism in the regulation of SV fusion and in the endocytic recycling of SV membranes (McMahon et al., 2010). The results reported here extend the role of MC to the reversible association of Syn with SV membranes, and thus, to the Syn-dependent organization of the RP (Pieribone et al., 1995; Rosahl et al., 1995; Ryan et al., 1996; Cesca et al., 2010). Since Syn I undergoes cycles of activity-dependent phosphorylation and concomitant SV association/dissociation (Cesca et al., 2010), it is tempting to speculate that ALPS-mediated curvature sensing by Syn I might be modulated by phosphorylation-induced conformational changes.

Interestingly, $\alpha$-synuclein, a presynaptic SV-associated protein, also senses MC (Varkey et al., 2010; Pranke et al., 2011), and its overexpression inhibits neurotransmitter release due to defects in SV reclustering (Nemani et al., 2010), similar to loss of Syn I (Rosahl et al., 1995; Ryan et al., 1996). Together with our findings reported here, these results are consistent with the hypothesis that SV clustering is regulated by curvature sensors on the SV membrane, potentially regulating SV pool sizes, synaptic transmission, and short-term plasticity.

\section{References}

Antonny B (2011) Mechanisms of membrane curvature sensing. Annu Rev Biochem 80:101-123.

Benfenati F, Greengard P, Brunner J, Bähler M (1989) Electrostatic and hydrophobic interactions of synapsin I with phospholipid vesicles. J Cell Biol 108:1851-1862.

Benfenati F, Valtorta F, Rossi MC, Onofri F, Sihra T, Greengard P (1993) Interactions of synapsin I with phospholipids: possible role in synaptic vesicle clustering and in the maintenance of bilayer structures. J Cell Biol 123:1845-1855.

Bhatia VK, Madsen KL, Bolinger PY, Kunding A, Hedegård P, Gether U, Stamou D (2009) Amphipathic motifs in BAR domains are essential for membrane curvature sensing. EMBO J 28:3303-3314.

Bigay J, Casella JF, Drin G, Mesmin B, Antonny B (2005) ArfGAP1 responds to membrane curvature through the folding of a lipid packing sensor motif. EMBO J 24:2244-2253.

Cesca F, Baldelli P, Valtorta F, Benfenati F (2010) The synapsins: key actors of synapse function and plasticity. Prog Neurobiol 91:313-348.

Chi P, Greengard P, Ryan TA (2001) Synapsin dispersion and reclustering during synaptic activity. Nat Neurosci 4:1187-1193.

Drin G, Casella JF, Gautier R, Boehmer T, Schwartz TU, Antonny B (2007) A general amphipathic alpha-helical motif for sensing membrane curvature. Nat Struct Mol Biol 14:138-146.

Gitler D, Xu Y, Kao HT, Lin D, Lim S, Feng J, Greengard P, Augustine GJ (2004) Molecular determinants of synapsin targeting to presynaptic terminals. J Neurosci 24:3711-3720.

Hosaka M, Südhof TC (1999) Homo- and heterodimerization of synapsins. J Biol Chem 274:16747-16753.

McMahon HT, Kozlov MM, Martens S (2010) Membrane curvature in synaptic vesicle fusion and beyond. Cell 140:601-605.

Menegon A, Bonanomi D, Albertinazzi C, Lotti F, Ferrari G, Kao HT, Benfenati F, Baldelli P, Valtorta F (2006) Protein kinase A-mediated synapsin I phosphorylation is a central modulator of $\mathrm{Ca}^{2+}$-dependent synaptic activity. J Neurosci 26:11670-11681.

Monaldi I, Vassalli M, Bachi A, Giovedì S, Millo E, Valtorta F, Raiteri R, Benfenati F, Fassio A (2010) The highly conserved synapsin domain E mediates synapsin dimerization and vesicle clustering. Biochem J 426:55-64.

Murthy VN, De Camilli P (2003) Cell biology of the presynaptic terminal. Annu Rev Neurosci 26:701-728.

Nemani VM, Lu W, Berge V, Nakamura K, Onoa B, Lee MK, Chaudhry FA, Nicoll RA, Edwards RH (2010) Increased expression of alpha-synuclein reduces neurotransmitter release by inhibiting synaptic vesicle reclustering after endocytosis. Neuron 65:66-79.

Pieribone VA, Shupliakov O, Brodin L, Hilfiker-Rothenfluh S, Czernik AJ, Greengard P (1995) Distinct pools of synaptic vesicles in neurotransmitter release. Nature 375:493-497.

Pranke IM, Morello V, Bigay J, Gibson K, Verbavatz JM, Antonny B, Jackson CL (2011) Alpha-synuclein and ALPS motifs are membrane curvature sensors whose contrasting chemistry mediates selective vesicle binding. J Cell Biol 194:89-103.

Rizzoli SO, Betz WJ (2005) Synaptic vesicle pools. Nat Rev Neurosci 6:57-69.

Rosahl TW, Spillane D, Missler M, Herz J, Selig DK, Wolff JR, Hammer RE, Malenka RC, Südhof TC (1995) Essential functions of synapsins I and II in synaptic vesicle regulation. Nature 375:488-493.

Ryan TA, Li L, Chin LS, Greengard P, Smith SJ (1996) Synaptic vesicle recycling in synapsin I knock-out mice. J Cell Biol 134:1219-1227.

Schiebler W, Jahn R, Doucet JP, Rothlein J, Greengard P (1986) Characterization of synapsin I binding to small synaptic vesicles. J Biol Chem 261:8383-8390.

Shupliakov O, Haucke V, Pechstein A (2011) How synapsin I may cluster synaptic vesicles. Semin Cell Dev Biol 22:393-399.

Takamori S, Holt M, Stenius K, Lemke EA, Grønborg M, Riedel D, Urlaub H, Schenck S, Brügger B, Ringler P, Müller SA, Rammner B, Gräter F, Hub JS, De Groot BL, Mieskes G, Moriyama Y, Klingauf J, Grubmüller H, Heuser J, et al. (2006) Molecular anatomy of a trafficking organelle. Cell 127:831-846.

Varkey J, Isas JM, Mizuno N, Jensen MB, Bhatia VK, Jao CC, Petrlova J, Voss JC, Stamou DG, Steven AC, Langen R (2010) Membrane curvature induction and tubulation are common features of synucleins and apolipoproteins. J Biol Chem 285:32486-32493. 